\title{
Efficacy of ultrasonic mechanical instrumentation with and without chlorhexidine in treatment of chronic periodontitis
}

\author{
Keshav A. \\ Associate Professor, Dept. of Periodontology, School of Dental Science, Krishna Institute of Medical Sciences, Deemed to be \\ University, Karad, Maharashtra, India \\ *Corresponding Author: \\ Email: dr_keshav16@yahoo.co.in
}

\begin{abstract}
Aim: The aim of the present study was to evaluate the effects of ultrasonic mechanical instrumentation (UMI) along with the professional use of chlorhexidine (CHX) compared with UMI alone during periodontal supportive therapy in patients with chronic periodontitis.

Material and Methods: A total of twenty patients were included in the study and were divided into test and control group. The test group (ten patients) was treated with a single session of UMI along with subgingival irrigation under cavitation with $\mathrm{CHX}$ $0.02 \%$. A $0.2 \% \mathrm{CHX}$ solution was used for professional tongue brushing and mouth rinsing. The control group (ten patients) received a similar single session of UMI along with subgingival irrigation and professional tongue brushing and mouth rinsing with a control formulation. Clinical and microbiological parameters were assessed pre-treatment at 3 and 6 weeks posttreatment.

Results: Results showed that there was a significant reduction of supragingival plaque and gingival inflammation as well as a significant reduction of subgingival bacterial pathogens in both test and control groups. The additional use of CHX did not result in any additional clinical and microbiological benefit with respect to UMI alone.

Conclusion: CHX formulations in conjunction with UMI seems to produce no additional benefits over UMI alone during supportive therapy in chronic periodontitis patients.
\end{abstract}

Keywords: Chlorhexidine, Chronic Periodontitis, Piezoelectric Ultrasonic, Subgingival Microbiota.

\section{Introduction}

Periodontitis is a chronic inflammatory disease of tooth and supporting structures with clinical signs of bone and connective tissue loss and is mediated by a combination of periodontal pathogens and host defense systems. The main treatment of periodontal disease is nonsurgical treatment by scaling and root planing, which has been confirmed as a gold standard treatment of periodontitis. However, some patients may not respond favorably to nonsurgical treatment, which might be due to recolonization and reinfection with microorganisms remaining in soft and hard tissues. Other reasons include difficulty of access to deep periodontal pockets, furcation areas and root concavities.

Several studies have reported significant clinical and microbiological improvement in patients with severe periodontitis who have been treated with standard scaling and root planing along with full mouth disinfection using chlorhexidine (CHX). ${ }^{1}$ Similarly evidence suggests that ultrasonic mechanical instrumentation (UMI) is an effective method for removing supra and subgingival bacterial biofilm adherent to tooth surfaces ${ }^{2,3}$ as well as reducing probing depths and bleeding on probing scores. ${ }^{4,5}$

Microbial studies evaluating the effects of UMI on dental plaque have shown that subgingival debridement to determine substantial changes in the microbial composition of dental biofilm. In particular, UMI, results in reduced total bacterial count (TBC) while establishing a subgingival microflora consistent with periodontal healthy. ${ }^{7}$ Unfortunately, the microbial shift after periodontal debridement may be transient, and bacterial re-colonization of the root surface by pathogenic bacteria, which frequently occurs after treatment, may lead to disease recurrence. . $^{8-10}$

The presence of various bacterial reservoirs in the oral cavity, such as the tongue, tonsils, periodontal sulcus/pockets and exposed roots, seems to increase the likelihood of re-infection following treatment. ${ }^{12}$ In this respect, combined treatment with manual and/or mechanical instrumentation associated with local application of antimicrobial agents has been investigated widely. Recently, a systematic review suggested that the adjunctive subgingival irrigation with CHX, hydrogen peroxide or saline, in conjunction with periodontal debridement, offered limited advantages when compared with periodontal debridement alone. ${ }^{13}$

In contrast, when professional supra-and subgingival instrumentation is associated with a more comprehensive antimicrobial regimen, based on extensive professional and home-based use of antimicrobial agents according to the 'full-mouth disinfection' protocol, additional benefits on a positive shift of subgingival microbiota may be expected. ${ }^{14}$ Data seem to indicate that one-stage; full-dentition periodontal debridement with the adjunctive use of antimicrobials may be a key factor for the observed clinical and microbiological benefits over a classical stepwise periodontal debridement, at least for nonsurgical treatment of periodontally affected patients. ${ }^{5,16,17}$ 
The aim of the present randomized, double-blind, controlled study was to evaluate the clinical and microbiological effects of UMI associated with the professional use of CHX containing formulations compared with UMI alone during a recall session of periodontal supportive therapy in patients with chronic periodontics.

\section{Material and Methods}

Twenty systemically healthy chronic periodontitis patients were selected for study and were divided into test and control group. To be enrolled in the study, patients had to fulfill the following inclusion criteria: a clinical diagnosis of chronic periodontitis at the initial visit and patients willing to provide informed consent and to ensure compliance throughout the study. All patients were clinically healthy, except for the presence of periodontitis.

The exclusion criteria included: Pregnancy or lactation; physical or mental handicap; systemic and/or topical steroidal and non-steroidal anti-inflammatory drugs, systemic and/or topical antibiotics during the last 6 weeks before the study; fixed or removable orthodontic devices; oral soft tissue pathology.

Before entering the experimental phase, patients were given oral and written information on the study design/aim and effects of antimicrobial agents in order to have signed consensus. The study design was approved by the local ethical committee.

Antimicrobial formulations: In the present study, a test and a control formulation were used. The test formulation consisted of $0.2 \% \mathrm{CHX}$ solution and control formulation consisted of normal saline. The formulations that were prepared resembled identical without being distinguished between each other but the bottles were coded in order to differentiate. This resulted in a doubleblind study, so that neither the patient nor the investigator was aware of which treatment had been assigned.

Treatment regimen: The treatment regimen for test and control group varied in the subgingival irrigation which was a CHX formulation for test goup and a control formulation for control group. At baseline, the test group received a single session of full-mouth supra and subgingival mechanical instrumentation by means of a piezoelectric ultrasonic device followed by supragingival polishing with a non-fluoridated prophylaxis paste. The ultrasonic instrumentation was associated with subgingival irrigation under cavitation with $0.2 \% \mathrm{CHX}$ formulation diluted 1:10 with saline (i.e. $0.02 \%$ CHX). Along with mechanical debridement professional brushing on the dorsum of the tongue using $0.2 \% \mathrm{CHX}$ was performed and the patient was also asked to gargle with $0.2 \% \mathrm{CHX}$.

The control group received a similar session of mechanical debridement associated with subgingival irrigation under cavitation with normal saline. Tongue brushing and mouth rinsing were also performed.
Experimental design: The present study was a randomized, controlled, double-blind study. The patients were assigned to either test and control group based on the type of formulation used in that particular individuals. The examiners were kept blind to allocation sequence and were unaware of formulations. At week 0 (baseline), the patients were assigned to either the test or the control formulation. Clinical recordings and subgingival plaque samples were collected. Verbal oral hygiene instructions $(\mathrm{OHI})$ on mechanical plaque control were given. At week 3 and 6 following the session, clinical parameters and subgingival plaque samples were recorded. OHI were reinforced. At week 6, periodontal debridement and prophylaxis were provided as needed for plaque/calculus/stain elimination.

Clinical recordings: Clinical parameters were recorded at weeks 0,3 and 6 which included:

Gingival index (GI), according to Loe \& Silness (1963). Presence of supragingival plaque according to Plaque index (PI; Turesky et al. 1970).

Pocket probing depth (PPD) using Williams graduated periodontal probe on all teeth. A disclosing agent was used to detect plaque.

PI and GI were recorded at six sites (mesio-buccal, buccal, disto-buccal, mesiolingual, lingual and distolingual) on the following selected teeth: \#16, \#11, \#26, \#36, \#31 and \#46. If one of these teeth was missing, the available adjacent tooth was examined.

Microbiological samples: Microbial plaque samples were collected from the subgingival area using sterile paper points. One site was selected from each quadrant for sample collection where the probing depth was > $5 \mathrm{~mm}$. Sample collection was performed by isolating the area with cotton rolls and the area was dried with air. The samples were collected by an investigator who was blinded to treatment regimen at weeks 0 (immediately before instrumentation), 3 and 6 . A sterile paper point was inserted subgingivally into each site and left in place for $20 \mathrm{~s}$. Then, the samples were pooled, immediately transferred to a sterile transport tube and sent to a laboratory. Polymerase Chain Reaction (PCR) was used to know the total bacterial count (TBC), as well as for detection of bacteria such as Porphyromonas gingivalis (P.g),Treponema denticola (T.d), Fusobacterium nucleatum (F.n), Prevotella intermedia (P.i) and Tannerella forsythia (T.f). The level of detection was set at $102 \mathrm{bacteria/plaque}$ sample. The sampling sites were kept constant throughout the study.

\section{Statistical analysis}

Statistical analysis was performed using the obtained data. Mann-Whitney test was performed for data from clinical parameters (i.e. GI, PI and PPD) to explore the inter-treatment differences and were expressed as median. The four pooled paper points, as collected at each observation interval, were processed together to give patient based bacterial counts. Before the analysis, bacterial counts were transformed to 
logarithms (base 10). The total number of pathogens (i.e P.g, T.d, F.n, P.i, and T.f) were added together to obtain the total pathogen (TP) count. The level of significance was set at $5 \%$. The minimum benefit, in terms of reduction of the baseline value with respect to 6 weeks, that would be needed (with $80 \%$ statistical power and $\mathrm{a}=0.05$ ) for groups to show a statistical difference was calculated: 0.3 for PI reduction; 0.2 for GI reduction; and (log base 10) for TBC.

\section{Results}

Of all the 20 chronic periodontitis patients who had been enrolled the results were as follows:

Supragingival plaque accumulation: The data that was analyzed using statistical analysis was tabulated. Table 1 depicts the PI scores as recorded in the test and control groups at each observation interval. In both the test and the control groups, a statistically significant decrease in PI was observed during the experimental phase $(p=$ 0.002 for the test group and $\mathrm{p}=0.001$ for the control group). Baseline values were significantly higher compared with the 3 , and 6-week values $(\mathrm{p}<0.05)$. Though there was a gradual reduction in plaque scores but no significant differences in PI were observed between the test and control groups at any observation intervals.

Table 1: Plaque index (PI) in control $(n=10)$ and test $(n=10)$ group assessed at six selected teeth (median)

\begin{tabular}{|l|c|c|c|}
\hline \multicolumn{1}{|c|}{} & Control - PI & Test - PI & \multirow{2}{*}{$\begin{array}{c}\text { p- } \\
\text { value* }\end{array}$} \\
\cline { 2 - 3 } & Median & Median & value \\
\hline Baseline & 2.8 & 2.70 & 0.123 \\
\hline 3 weeks & 1.10 & 1.25 & 0.89 \\
\hline 6 weeks & 1.80 & 1.75 & 0.130 \\
\hline
\end{tabular}

* Mann-Whitney test p-value to compare between test and control groups at each observational interval.

Gingival inflammation: Table 2 shows the GI scores as analyzed for the test and control groups. GI baseline values were significantly higher compared with the 3 and 6 week values $(p<0.05)$. Even GI scores significantly decreased in the test and control groups ( $p=0.02$ for the test group, $\mathrm{p}=0.00$ for the control group), but no significant differences in GI were observed between the test and control groups at any observation interval.

PPD: Table 3 shows the descriptive statistical analysis of PPD, which revealed that even PPD significantly decreased over time in the test $(\mathrm{p}=0.005)$ and control groups $(\mathrm{p}<0.001)$.There was a gradual decrease in PPD in both groups from baseline to 3 weeks and remained stable thereafter. No significant differences in PPD were observed between the test and control groups at any observation interval.
Table 2: Gingival index (GI) in control $(n=10)$ and test $(n=10)$ group assessed at six selected teeth (median)

\begin{tabular}{|l|c|c|c|}
\hline & Control - GI & Test - GI & $\begin{array}{c}\text { p- } \\
\text { value* }\end{array}$ \\
\cline { 2 - 4 } & Median & Median & 0.85 \\
\hline Baseline & 1.50 & 1.20 & 0.75 \\
\hline 3 weeks & 0.90 & 0.80 & 0.23 \\
\hline 6 weeks & 1.10 & 0.70 & 0.00 \\
\hline
\end{tabular}

* Mann-Whitney test p-value to compare between test and control groups at each observational interval

Table 3: Probing depth (P PD) in control $(n=10)$ and test $(n=10)$ group assessed at six selected teeth (median)

\begin{tabular}{|c|c|c|c|}
\hline & Control -PPD & Test - PPD & P. \\
\hline & Median & Median & value* \\
\hline Baseline & 4.75 & 4.90 & 0.32 \\
\hline 3 weeks & 3.90 & 4.0 & 0.42 \\
\hline 6 weeks & 4.10 & 4.20 & 0.53 \\
\hline
\end{tabular}

*Mann-Whitney test p-value to compare between test and control groups at each observational interval.

PCR analysis (TBC and TP): TBC significantly decreased over time in the control group $(p=0.002)$. In the test group, TBC showed a marked reduction over time; however, this change did not reach statistical significance $(\mathrm{p}=0.07)$. TBC significantly decreased from baseline to 3 weeks and remained similarly low thereafter in both groups. No significant differences in TBC were observed between the test and control groups at any observation interval (Fig 1). Similarly TP was reduced in both test and controls at 3 and 6 weeks when compared to baseline but this reduction in TP did not show any significant differences at different intervals between test and controls $(\mathrm{p}=0.005$ and $\mathrm{p}<0.001$ respectively) (Fig 2).

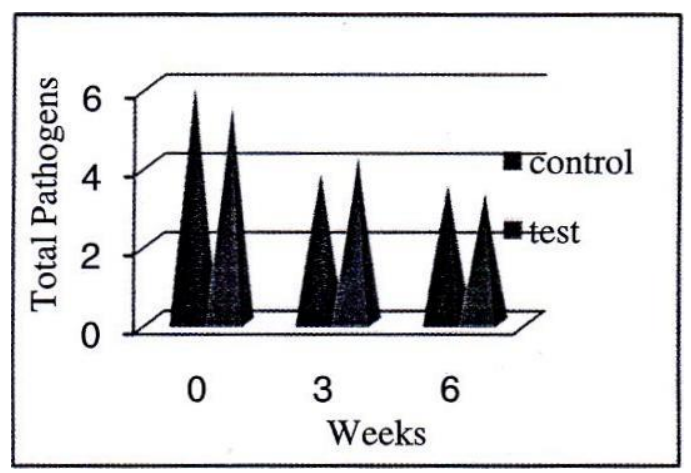

Fig. 1: Total bacterial count (Logarithm Base 10) 


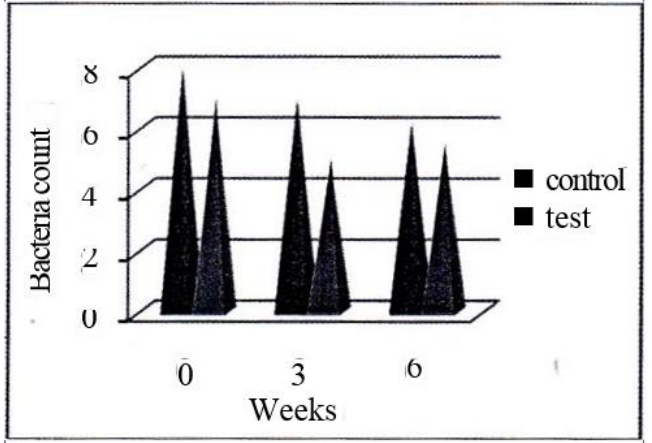

Fig. 2: Total pathogens count (Logarithm Base 10)

\section{Discussion}

In the present randomized double blind clinicomicrobial study, we evaluated the efficacy of professional use of CHX formulation with single session of UMI compared with UMI alone during supportive periodontal therapy in patients with chronic periodontitis. Clinical and microbiological parameters were assessed at observational intervals of 0 weeks (i.e. baseline), 3 and 6 weeks following the UMI session. Clinical parameters included PI, GI and PPD, whereas microbial samples consisted of subgingival plaque samples collected at four sites, one for each quadrant, using paper points. The sites for microbial sample collection were chosen at the initial appointment (i.e. baseline) and were kept constant as same sites throughout the study.

In the present study design a piezoelectric ultrasonic device was used for debridement of supra and subgingival deposits, which was followed by supragingival polishing done using a non-fluoridated prophylaxis paste. A diluted $0.2 \% \mathrm{CHX}$ was used as an irrigating agent during UMI in the test group along with which a $0.2 \% \mathrm{CHX}$ solution was used for oral rinsing and tongue brushing. The control group received normal saline for irrigation and rinsing. The assumption was that the cleaning efficacy of ultrasonic scaling in removing plaque and calculus would have been implemented by the antimicrobial effect of CHX. In turn, plaque removal and bacterial cell disruption through the vibrating chipping action of the tip, the cavitational activity and the acoustic micro streaming due to the ultrasonic scaler would have improved the bactericidal effect of the CHX, leading to a greater reduction of subgingival microflora18. Moreover, CHX for mouthrinse and tongue brushing aimed to enhance the suppression of periopathogens from all their oro-pharyngeal habitats, thus potentially delaying the re-colonization (by infraoral translocation) of the treated pockets by bacteria from untreated sites 9 . All the way throughout the entire study (6-week observation interval) both the test and the control treatment resulted in a marked reduction of approximately one $\log \mathrm{TBC} /$ plaque sample with respect to the baseline values (median log reduction: test group: 0.78, control group: 0.86).
The use of $\mathrm{CHX}$ as a subgingival irrigant along with mechanical debridement has variable outcomes and this can be attributed to the type of study conducted i.e based on concentration and dose of CHX used mechanical debridement whether done with SRP or with ultrasonic instrumentation or it could be due to frequency of irrigation and type of irrigating device $2^{\circ} .{ }^{22}$

In the present study both the test and control group showed that PI markedly reduced from baseline to 3 weeks and was maintained constantly below the baseline values during the 6 week interval. Similarly GI was also reduced substantially from baseline to 3 weeks and remained the same till 6 weeks. The lack of effects of adjunctive $\mathrm{CHX}$ in the present study could or may be due to low concentration of CHX i.e. $0.02 \%$. Similar results were obtained with no significant differences in microbiological and clinical parameters in studies when identical CHX concentrations were used in conjunction with ultrasonic mechanical instrumentation. ${ }^{23}$ However, even when higher concentrations of CHX (0.12-0.2\%) were used as a coolant during ultrasonic scaling, the effect on clinical parameters and subgingival microflora appeared to be limited, if any. ${ }^{24}$ Thus with these evidences we can draw and assume that the thoroughness of plaque removal can be much effective with ultrasonic mechanical instrumentation when compared with the action of antimicrobial chemical agent.

Microbial analysis was done using PCR to know the TBC which revealed a marked reduction at 3 weeks following treatment and did not revert to the original values at 6 weeks in both the test and the control group. Parallel to the microbial shift, a significant reduction in PPD from baseline values $(>5 \mathrm{~mm})$ was observed at microbiologically sampled sites. Similar results have been reported between a low proportion of periodontal pathogens and a reduction in pocket depth and gain in attachment level following periodontal treatment. ${ }^{25,26}$

Professional removal of supragingival plaque, combined with careful self-performed plaque control, may induce marked qualitative and quantitative alterations of the subgingival microbiota in subjects with periodontitis and moderately deep pockets. ${ }^{27-29}$ It has been shown that the formation of subgingival dental biofilm is closely related to the accumulation of supragingival plaque deposits..$^{30,31}$ Recolonization of the subgingival area may also have been affected by the strict plaque control regimen adopted by the patients. The adjunctive self-performed mechanical plaque control may also have reduced the contribution of bacteria present in other oral ecological niches to subgingival recolonization. ${ }^{32,33}$ Thus the results support the use of stringent oral hygiene protocols supplemented by the use of antimicrobial agents to control early subgingival re-colonization by periodontal pathogens, particularly in patients with high susceptibility to destructive periodontal disease. 


\section{Conclusion}

The results of the present study indicates that CHX when used as an adjunctive to mechanical instrumentation did not show any beneficial effects either on clinical or microbiological parameters during supportive periodontal therapy in chronic periodontitis patients. However, such results when compared with other studies which have shown positive response of CHX on clinical and microbiological parameters draw a very controversial conclusion. But results as in the present study could be due to the limited sample size and the specificity of the study population, further randomized controlled trials need to be conducted to confirm these preliminary findings in a broader population affected by different forms of periodontal diseases.

Funding: No funding sources.

Conflict of interest: None declared.

\section{References}

1. Quirynen M, Mongardini C, de Soete M, Pauwels M, Coucke W, Van Eldere J, Van Steenberghe D. The role of chlorhexidine in the one-stage full-mouth disinfection treatment of patients with advanced adult periodontitis. Longterm clinical and microbiological observations. $J$ Clin Periodontol 2000;27:578-89.

2. Thornton S, Garrick J. Comparison of ultrasonic to hand instruments in the removal of subgingival plaque. $J$ Periodontol 1982;53:35-7.

3. Breininger DR, O'Leary TJ, Blumenshire RVH. Comparative effectiveness of ultrasonic and hand scaling for the removal of subgingival plaque and calculus. $J$ Periodontol 1987;58:9-18.

4. Greenstein G. Nonsurgical periodontal therapy in 2000: a literature review. J Am Dent Assoc 2000;131:1580-92.

5. Drisko $\mathrm{CH}$. Non surgical periodontal therapy. Periodontol 2000,2001;25:77-88.

6. Oosterwaal PJM, Matee MI, Mickx FHM, van't Hof MA, Renggli HH. The effect of subgingival debridement with hand and ultrasonic instruments on the subgingival microflora. J Clin Periodontol 1987;14:528-33.

7. Baehni P, Thilo B, Chapuis B, Pernet D. Effects of ultrasonic and sonic scalers on dental plaque microflora in vitro and in vivo. J Clin Periodontal 1992;19:455-59.

8. Mousque's T, Listgarten MA, Phillips RW. Effect of scaling and root planning on the composition of the human subgingival microbial flora. J Periodontal Res 1980;15:144-51.

9. Magnusson I, Lindhe J, Yoneyama T, Liljenberg B. Recolonization of a subgingival micribiota following scaling in deep pockets. J Clin Periodontol 1984;11:193207.Van Winkelhoff, A. J., van der Velden, U, De Graff, J. Microbial succession in recolonizing deep periodontal pockets after a single course of supra- and subgingival debridement. J Clin Periodontol 1998: 15; 116-122.

10. Van der Velden, U., Van Winkelhoff, A. J., Abbas, F. \& De Graaff, J. The habitat of periodontopathic microorganism. J Clin Periodontol 1986;32:243-48.

11. Adriaens P, Edwards C, Deboever J, Loesche W. Ultrastructural observations on bacterial invasion in cementum and radicular dentine of periodontally diseased human teeth. J Periodontol 1988;59:493-503.
12. Hallmon W, Rees TD. Local antiinfective therapy: mechanical and physical approaches. A systematic review. Ann Periodontol 2003;8:99-114.

13. Bollen CML, Mongardini C, Papaioannou W, van Steenberghe D, Quirynen M. The effect of one-stage fullmouth disinfection on different intra-oral niches. Clinical and microbiological observation. J Clin Periodontol 1998;25:56-66.

14. Quirynen M, Bollen CML, Vandekerkhove BNA, Dekeyser C, Papaioannou W, Eyssen H. Full-vs. Partial mouth disinfection in the treatment of periodontal infections: short-term clinical and microbiological observations. J Dent Res 1995;74:1459-67.

15. De Soete M, Mongardini C, Peuwels M, Haffajee A, Socransky S, van Steenberghe D, Quirynen M. One-stage full-mouth disinfection. Long-term microbiological results analyzed by checkboard DNA-DNA hybridization. J Periodontol 2001;72:374-82.

16. Wennstrom GL, Tomasi C, Bertelle A, Dellasega E. Fullmouth ultrasonic debridement versus quadrant scaling and root planing as an initial approach in the treatment. $J$ Clin Periodontol 2005;32(8):851-59.

17. Walmsley AD, Laird WRE, Williams AR. A model system to demonstrate the role of cavitational activity during ultrasonic scaling. J Dent Res 1984;63:1162-65.

18. Faveri M, Gursky LC, Feres M, Shibly JA, Salvador SL, de Figueiredo LC. Scaling and root planing and chlorhexidine in the treatment of chronic periodontitis: a randomized, placebo-controlled clinical trial. J Clin Periodontol 2006;33:819-28.

19. Watts EA, Newman HN. Clinical effects on chronic periodontitis of a simplified system of oral hygiene including subgingival pulsated jet irrigation with chlorhexidine. J Clin Periodontol 1986;13:666-70.

20. Reynolds MA, Lavigne CH, Minah GE, Suzuchi JB. Clinical effects of simultaneous ultrasonic scaling and subgingival irrigation with chlorhexidine. Mediating influence of periodontal probing depth. J Clin Periodontol 1992;19:595- 600.

21. Bollen CML, Quirynen, M. Microbiological response to mechanical treatment in combination with adjunctive therapy. A review of the literature. J Periodontol 1996;67:1143-58.

22. Taggart JA, Palmer RM, Wilson RF. A clinical and microbiological comparison of the effects of water and $0.02 \%$ chlorhexidine as coolants during ultrasonic scaling and root planing. J Clin Periodontol 1990;17:32-7.

23. Chapple IL, Walmsley AD, Saxby MS, Moscrop H. Effect of subgingival irrigation with chlorhexidine during ultrasonic scaling. J Periodontol 1992;63:812-69.

24. Van Winkelhoff AJ, van der Velden U, De Graff J. Microbial succession in recolonizing deep periodontal pockets after a single course of supra- and subgingival debridement. J Clin Periodontol 1988;15:116-22.

25. Haffajee AD, Cugini MA, Dibart S, Smith C, Kent RL, Socransky SS. The effect of SRP on the clinical and microbiological parameters of periodontal disease. J Clin Periodontol 1997;24:324-34.

26. Tabita PV, Bissada NF, Maybury GE. Effectiveness of supragingival plaque control on the development of subgingival plaque and gingival inflammation in patients with moderate pocket depth. J Periodontol 1981;52:8893.

27. Dahle'n G, Lindhe J, Sato K, Hanamura H, Okamoto H. The effect of supragingival plaque control on the subgingival microbiota in subjects with periodontal disease. J Clin Periodontol 1992;19:802-09. 
28. Hellstro-m MK, Ramberg P, Krok L, Lindhe J. The effect of supragingival plaque control on the subgingival microflora in human periodontitis. J Clin Periodontol 1996;23:934-40.

29. Haffajee AD, Arguello EI, XimenezFyvie LA, Socransky SS. Controlling the plaque biofilm. Int Dent J 2003;53: 191-99.

30. Tezal M, Scannapieco FA, Wactawski Wende J, Grossi SG, Genco RJ. Supragingival plaque may modify the effects of subgingival bacteria on attachment loss. $J$ Periodontol 2006;77:808-13.

31. Quirynen M, Bollen CML, Vandekerkhove BNA, Dekeyser C, Papaioannou W, Eyssen H. Full-vs. partial mouth disinfection in the treatment of periodontal infections: short-term clinical and microbiological observations. J Dent Res 1995;74:1459-67.

32. Bollen CML, Mongardini C, Papaioannou W, Van Steenberghe D, Quirynen M. The effect of a one-stage fullmouth disinfection on different intra-oral niches. Clinical and microbiological observation. J Clin Periodontol 1998;25:56-66.

How to cite the article: A. Keshav. Efficacy of ultrasonic mechanical instrumentation with and without chlorhexidine in treatment of chronic periodontitis. IP Int J Periodontol Implantol 2018;3(3):94-99. 